\title{
Enhancing Quality of Service in ERS- AODV for Mobile Ad Hoc Networks
}

\author{
Puneet Bindra \\ M.tech Scholar \\ SBSSTC, Ferozepur
}

\author{
Jaswinder Kaur \\ A.P (ECE) \\ SBSSTC, Ferozepur
}

\author{
Gurjeevan Singh \\ DIC (ECE) \\ SBSSTC(Polywing), Ferozepur
}

\begin{abstract}
A MANET is a wireless network which operates by themselves without centralized administration. Each node in MANET operates as an end system and a router for all other nodes in the network. The primary challenge in building a MANET is equipping each device to continuously maintain the information required to properly route the traffic. Reactive routing protocols are favoured in MANET's because they generate fewer overheads in network. These protocols suffer due to network flooding in route discovery process. Expanding ring search in terms of early detection of destination cause quality of service to fluctuate randomly. Performance degradation due to quality of service is a bottleneck to fulfill stability and reliability in MANET's. In this paper, a scheme is introduced for controlling the broadcasting of RREQ information focusing on improved quality of service in AODV (Ad hoc on Demand Distance Vector) routing protocol. This is achieved by monitoring the TTL start, TTL increment and TTL threshold values of network and choosing optimum values of TTL suitable to network designed that accounts for reliability and quality of service during route discovery process. It is simulated using OPNET 14.5 with fixed node density.
\end{abstract}

\section{Keywords}

\section{MANET, OPNET 14.5, Routing Protocol}

\section{INTRODUCTION}

MANET is a rapid growing technology from the past $20 \mathrm{yrs}$. MANET's created new set of demands to be implemented and to provide efficient better end to end communication [1]. MANET's are collection of mobile nodes equipped with wireless devices typically deployed in areas where no infrastructure exists. In MANET's users are active parts of network [2]. They dynamically join the network acting as both user terminals and routers for other devices. All nodes within a mobile ad hoc network use the same frequency band as a shared medium for receiving and transmitting data.

Reactive or on demand ad hoc routing protocols typically make use of request query broadcast to discover routes when a source to destination communication is needed. In AODV, the network is silent until a connection is needed. At that point the network node that needs a connection broadcast a request for connection. This protocol performs route discovery using route request (RREQ) and route reply (RREP) message whenever a node wants to sends a packet to destination. [3] Routing discovery of AODV is mainly divided into two parts, reverse route establishment and forward route establishment. In reverse route establishment, the source node broadcasts the RREQ packet firstly. When the destination node receives the
RREQ packet, it will send the route reply (RREP) packet to the source node, and then the route which the RREP packet passes through is called the reverse route. The forward route is established from the source node to the destination node which is used to transmit the following data packets. AODV uses expanding ring search technique for controlling broadcasting of RREQ messages. In this paper, TTL based expanding ring search for reliable and improved quality of service is modeled. The paper is structured as follows: a brief overview of the expanding ring search is given in Section 2. In Section 3 we present related work in ad hoc networks. Section 4 then enfolds Experimental methodology and Results and discussions are displayed in section 5 .Section 6 concludes this work and gives an outlook to further possible developments and research topics in this area.

\section{EXPANDING RING SEARCH}

Expanding Ring Search is used for making the route discovery process more efficient [4]. In the ERS scheme, the RREQ packet is broadcasted with a time to live (TTL) value. When the packet is received by other nodes, the TTL value on the packet is decremented. Then the packet is rebroadcasted with positive TTL value. This process continues until the destination is located or the TTL values reaches zero. In the latter case, a new query is transmitted by the source with a larger TTL value and the whole process is repeated. In some ERS techniques, the initial TTL value is set to 1 for the first ring search. If such a search fails to find the destination, a new search is initiated with incremented TTL value. The process continues until the initial TTL values reaches threshold. Then a network wide flooding is initiated.

In AODV routing protocol, an ERS scheme is implemented to start with TTL start and to increase the TTL by TTL increment after each failure. This technique works by searching successively larger areas in the network centered on the source of broadcast. [4]. In such a scheme the routing protocol performs several floodings with increasing TTL values, instead of a single network wide flood. TTL value is generally defined in route request messages which specify the number of times a particular route request message may be rebroadcast.

\section{RELATED WORK}

Existing approaches [5] include energy efficient route discovery process for AODV based on expanding ring search. In [6] impact of TTL sequence based ERS on energy aware routing protocols for MANETs is analyzed. New path updation and resolving link failure in AODV is done. [2] presents extensive studies of simulations for different routing protocols in homogeneous and heterogeneous networks that 
consist of different nodes with different resources. In [7] problem of intermediate route building in AODV has been solved that also enhances the performance of AODV protocol which consists of concept of local recovery with limited TTL value. Evaluation of effects of the route repair is done in [8] which are aimed to efficiently repair the link errors.

To control congestion by applying efficient local route repair method if destination was no farther than maximum repair TTL was proposed in [9]. In [10] efficient optimizations of bandwidth cost and energy is deduced by adjusting TTL value of network.

\section{EXPERIMEMTAL METHODOLOGY}

In this experiment, mobile ad hoc network consisting of 50, $100,150,200,250$ nodes randomly placed in 500 X 500 square meter area is generated and simulation duration is 200 seconds. The simulation tool used for analysis is OPNET 14.5 which is highly preferred by researchers [11]. It is a synthetic simulation tool to simulate and analyze for network communication and it can provide three tier model, where the top layer is the network layer that reflects the topology of the network, the middle layer is the node layer that is composed of the corresponding protocol models and the bottom layer is the process model that is described by finite state machines. The three tier model fully corresponds to the actual network, protocol and equipment. When OPNET is used to establish simulation models, all the necessary routing protocols are encapsulated in the IP layer.

Depending on total no. of nodes, size of network and diameter of network D, TTL values for expanding ring search are chosen as given in following Table1. For ERS1, ERS2, ERS3 TTL start and TTL threshold are constant and TTL increment is variable to evaluate the effect of early detection of destination.

Table 1. TTL Parameters

\begin{tabular}{|l|l|l|l|}
\hline ERS & $\begin{array}{l}\text { TTL } \\
\text { start }\end{array}$ & $\begin{array}{l}\text { TTL } \\
\text { increment }\end{array}$ & $\begin{array}{l}\text { TTL } \\
\text { threshold }\end{array}$ \\
\hline ERS1 & 1 & 1 & 15 \\
\hline ERS2 & 1 & 5 & 15 \\
\hline ERS3 & 1 & 9 & 15 \\
\hline
\end{tabular}

The simulation parameters used in experimental scenarios are shown in table 2 .

Table 2. Simulation Parameters

\begin{tabular}{|l|l|}
\hline Parameters & Value \\
\hline Simulation Area & $500 \mathrm{~m}$ X 500m \\
\hline Simulation Time & $200 \mathrm{sec}$ \\
\hline Mobile Node Placement & Random Way Point \\
\hline Buffer Size (BS) & 128000 \\
\hline Packet Size & 4096 bits \\
\hline Routing Protocol & AODV \\
\hline No. of Mobile Nodes(MN) & $50 / 100 / 150 / 200 / 250$ \\
\hline
\end{tabular}

\begin{tabular}{|l|l|}
\hline Transmit Power & $0.001 \mathrm{~W}$ \\
\hline Data rate & $12 \mathrm{Mbps}$ \\
\hline Node Speed & $2 / 4 / 8 / 16 \mathrm{~m} / \mathrm{s}$ \\
\hline Network Diameter & 25 \\
\hline Node Traversal Time & $0.06 \mathrm{sec}$ \\
\hline
\end{tabular}

The following metrics are evaluated for AODV routing protocol:

Delay: It represents end-to-end delay which refers to the time taken for a packet to be transmitted across a network from source to destination.

Packet Delivery Ratio: It is the ratio of the number of received packets at the destination to the number of packets that are sent by the source node. Route Discovery Time: The time taken from when a RREQ is assembled until the RREP is received. Number of Hops per Route: It represents no. of hops in each route to every destination in the route of all nodes in the network.

Total Route Requests Sent: It represents the total number of route requests packets sent by all node in the network during route discovery.

Total Replies Sent from Destination: It represents total number of route reply packets sent from all nodes in the network if they are destinations of route requests.

\section{RESULTS AND DISCUSSIONS}

In this section, simulation results have been discussed. The performance of route discovery process is analysed in terms of Delay, Packet Delivery Ratio, Route Discovery Time and Number of Hops per Route, Total Route Request Sent and Total Replies sent from Destination by evaluating effect of early detection of destination and by studying effects of node density and node speed on best chosen TTL value for expanding ring search.

\subsection{Effect of early detection of destination}

In this section, the performance of route discovery process is analyzed through different TTL values as basis for expanding ring search.

\subsubsection{Delay}

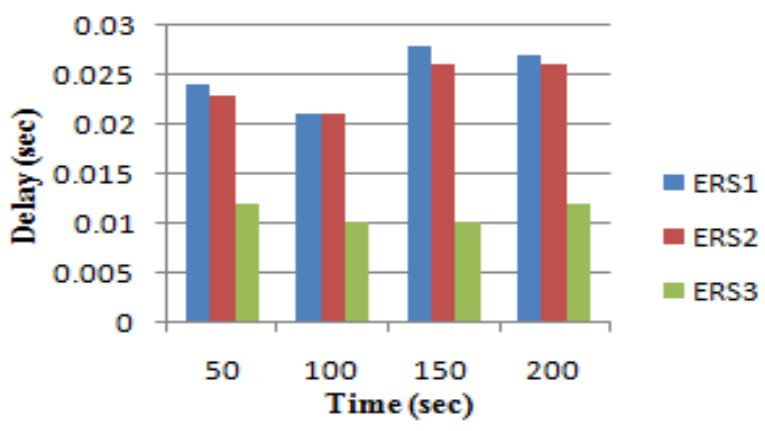

Fig 1: Delay

From figure 1 it is observed that ERS3 attains lowest routing latency in which TTL increment value is equal to 9 which results in quick searching of routes as number of route request 
propagating are less in ERS3 than ERS2 and ERS1 and results in early route discovery for network defined whereas ERS1 produces largest delay. ERS3 results with less delay as it has less number of route requests propagating per unit time.

\subsubsection{Packet Delivery Ratio}

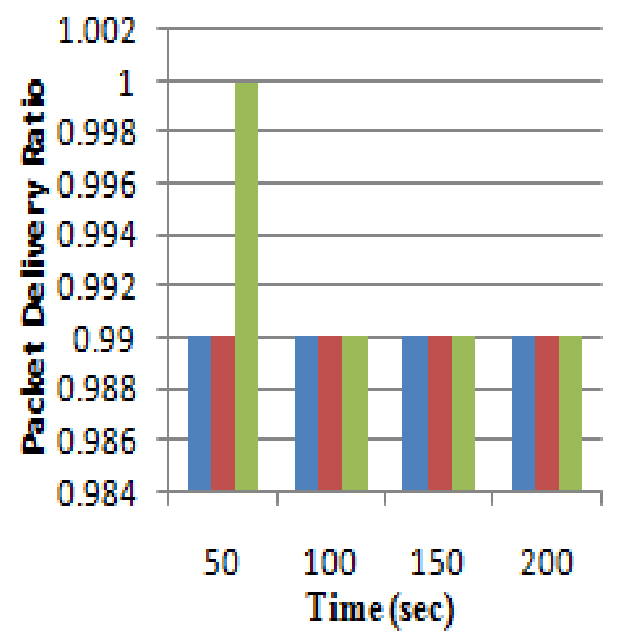

ERS1

Fig 2: Packet Delivery Ratio

Fig 2 illustrates the packet delivery ratio of expanding ring search with different TTL values. PDR of expanding ring search is nearly 0.99 for all TTL values (ERS1, ERS2 and ERS3) defined.

\subsubsection{Number of Hops per Route}

Number of hops per route is shown in figure 3. Average number of hops per route experienced is less in AODV ERS3 with greater TTL increment as the distance between source and destination for all nodes in the network would be less with more increment value.

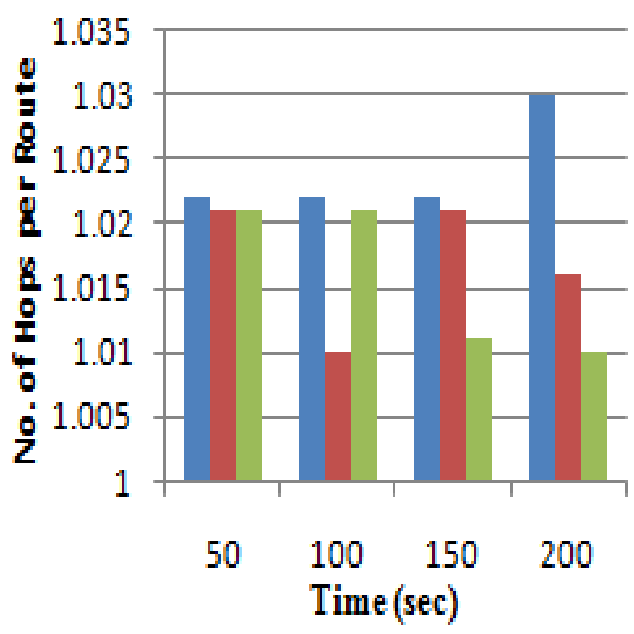

ERS1

Fig 3: Number of hops per route

\subsubsection{Route Discovery Time}

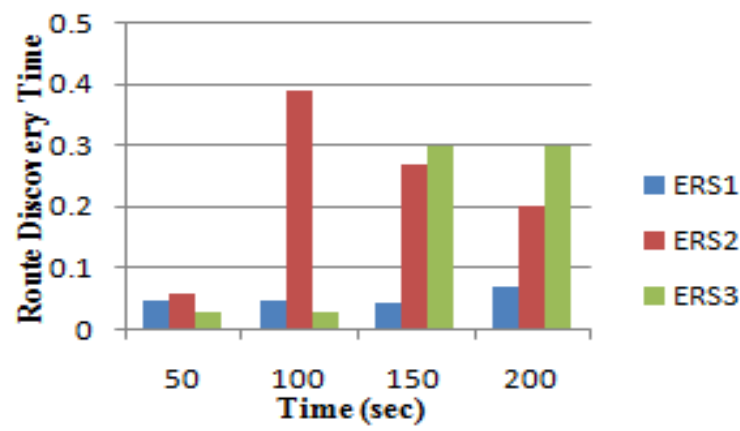

Fig 4: Route Discovery Time

Fig 4 shows effect of TTL increment on the route discovery time through expanding ring search. From fig it is observed that ERS3 tends to find the earliest path from the source node and destination node.ERS3 takes less route discovery time for some time and later route discovery time increases for same.

\subsubsection{Total Route Requests Sent}

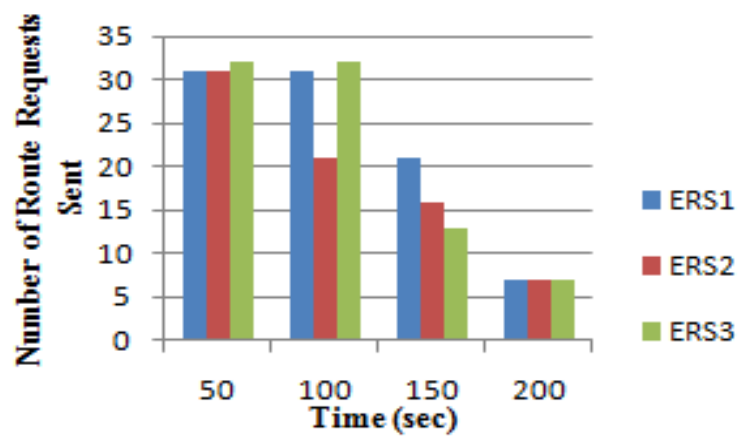

Total route requests sent are observed in fig 5 . Reduced number of RREQ is being transmitted by the nodes in ERS3 whereas ERS1 transmit more RREQ by the nodes. This is because of formation of more stable routes and active routes are less prone to route breakage in ERS3. Hence the numbers of control packets transmitted are least in ERS3.

\subsubsection{Total Route Replies Sent from Destination}

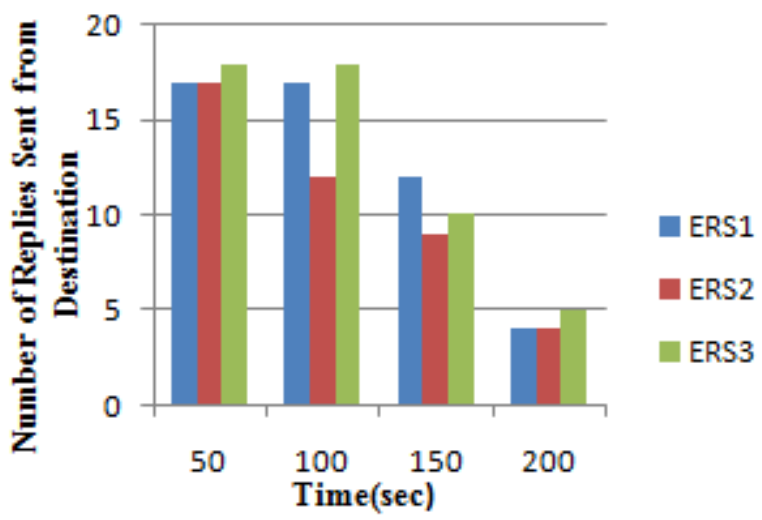

Fig 6: Total Route Replies Sent from Destination

From fig 6 it is clear that ERS3 consistently have more route replies sent from destination than ERS1 and ERS2 which is due to less probability of route errors and packets dropped in ERS3. 


\subsection{Effect of node density with respect to node speed on expanding Ring search}

In this section, effect of node density with respect to node speed on expanding ring search (ERS3) has been simulated as better results are experienced with ERS3. Simulations have been done for different number of nodes and for low and high mobility scenarios

\subsubsection{Delay}

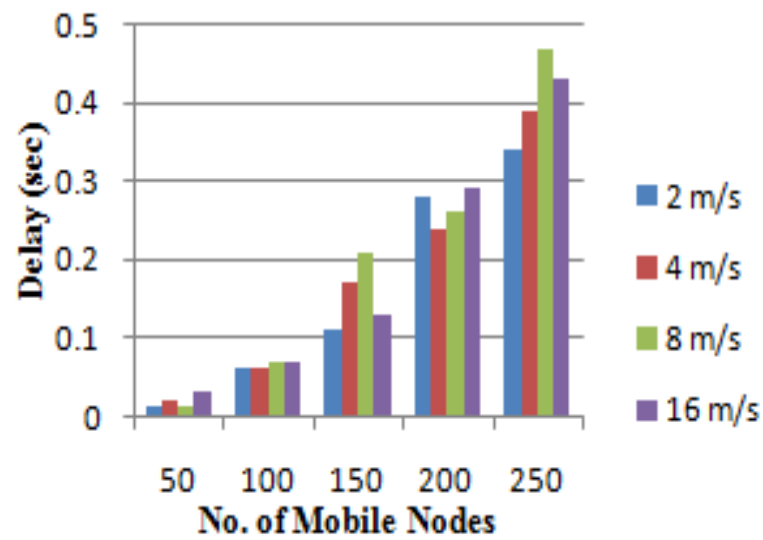

Fig 7: Delay- Varying nodes and node speed

Fig 7 shows delay with varying number of nodes and node speed. This metric is calculated by subtracting time at which first packet was transmitted by source from time at which the first data packet arrived to destination. Delay observed is least for least number of nodes and highest when number of nodes increases to 250. With increase in node speed there are significant changes observed as shown in fig.

\subsubsection{Packet Delivery Ratio}

Graphs show the fraction of data packets that are successfully delivered during simulation node speed versus number of nodes in fig 8. PDR is reducing regularly as the node speed and no. of nodes are increasing. Acc to given network parameters, PDR for least number of nodes versus node speed is better amongst all.

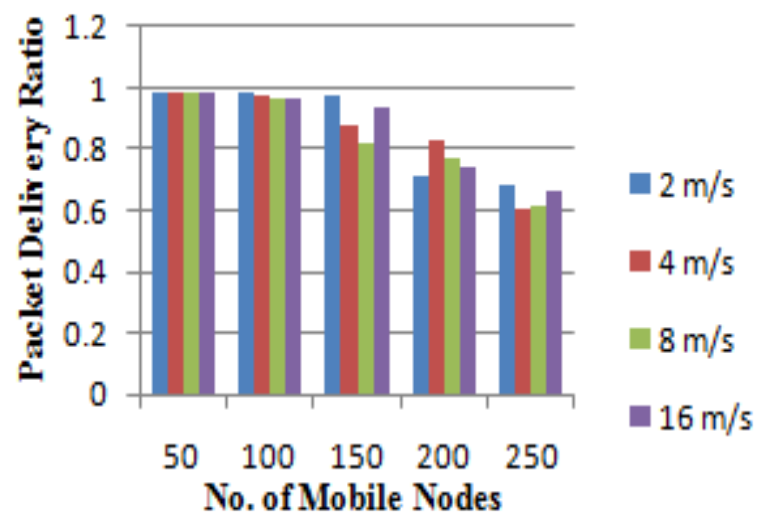

Fig 8: PDR- Varying nodes and node speed

\subsubsection{Number of Hops per Route}

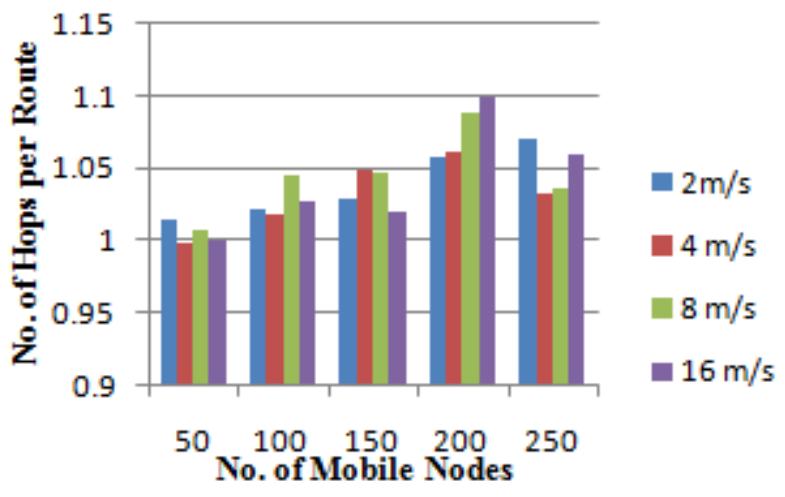

Fig 9: Hops per route- Varying nodes and node speed

Fig 9 shows number of hops per route with increasing number of nodes and node speed. The graph shows that all number of nodes behave differently with increase in mobility of nodes. Least number of hops per route is experienced with least number of nodes and least node mobility.

\subsubsection{Route Discovery Time}

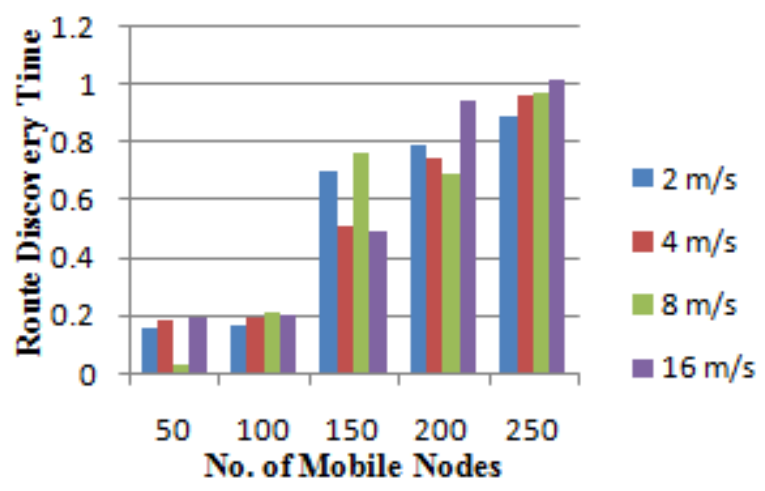

Fig 10: RDT- Varying nodes and node speed

Above graph shows variation of the route discovery time in fig 10. There is comparable increase in route discovery time as there is increase in node density and node mobility. Less time to discover route is taken by least number of nodes which is due to high probability to discover destination quickly.

\subsubsection{Total Route Requests Sent}

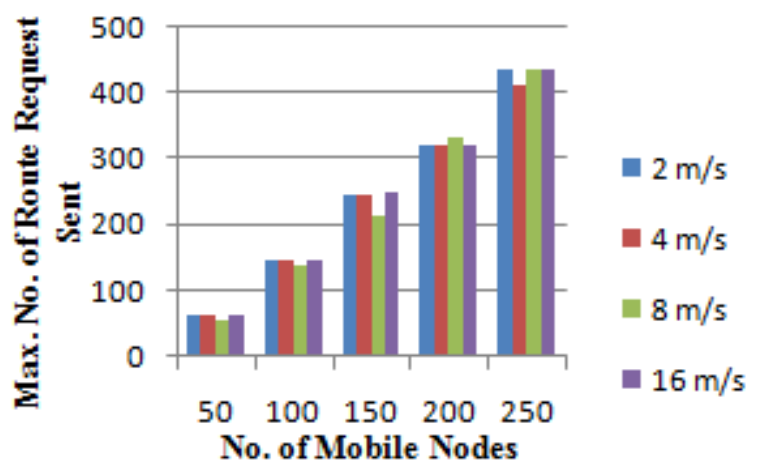

Fig 11: Route Requests- Varying nodes and node speed 
With the increase in number of nodes and node mobility, linear increase is observed in total route request sent by all nodes in the network as shown in fig 11. When nodes broadcast RREQ messages those messages are received by more nodes and each node receives a greater number of RREQ messages.

\subsubsection{Total Route Replies Sent from Destination}

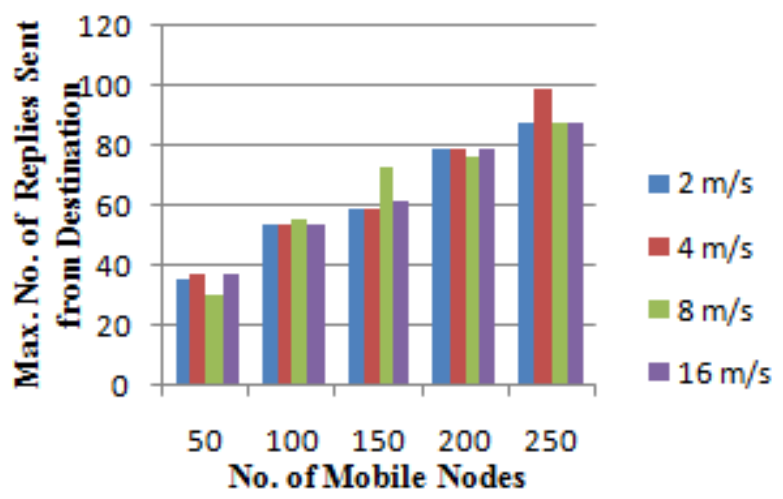

Fig 12: Route Replies-Varying nodes and node speed

Fig 12 shows total route replies sent from destination. As Route requests propagating increases as number of nodes and node mobility increases, total route replies sent from destination also increases with increase in node density and node mobility.

\section{CONCLUSION}

This paper presented expanding ring search proposed for improved quality of service and reliable route discovery. The proposed strategy significantly improves quality of service. Simulation results show ERS3 outperforms ERS1 and ERS2 in terms of delay, packet delivery ratio, number of hops per route, route discovery time, total route requests sent and total replies sent from destination. Based on ERS3 effect of node density and node speed is studied which shows Quality of service parameters and other performance characteristics increases with increase in node density but is independent of node speed.

\section{REFERENCES}

[1] Parulpreet Singh, Ekta Barkhodia and gurleen kaur walia," Evaluation of various Traffic loads in MANET with DSR routing protocol through use of OPNET Simulator" , IJDPS,Vol.3 No.3, pp 75-83, May 2012

[2] Huda Al Amri,Mehran Abolhasan and Tadeusz Wysocki," Scalability of MANET routing protocols for heterogeneous and homogeneous networks", Elsevier 2010

[3] Manijeh Keshtgary and Vahide Babaiyan," Performance Evaluation of Reactive, Proactive and Hybrid Routing Protocols in MANET", IJCSE, Vol. 4, pp 248-254, Feb 2012

[4] Purvi N.Raanuj and Hiteishi M. Diwanji," Enhanced Expanding Ring Search Algorithm for AODV”, IJCA, Jan 2012

[5] Rupali Mahajan and Rupali Jagtap,"Energy Efficient Routing Protocols for Mobile Ad hoc Networks", IJISME, Vol. 1 Issue 3, pp 27- 31, Feb 2013

[6] B.Prasad , Dr. S.P.Setty," Analysing the Impact of TTL Sequence based Expanding Ring Search on Energy Aware Routing Protocols for MANETs", IJEST, Vol.4 , pp $2197-$ 2205, May 2012

[7] Anurag Porwal et al," Study and Design of New Reactive Routing Protocol Advance AODV for Mobile Adhoc Networks", IJERA, Vol. 2 Issue 3, pp 3195-3204 May- June 2012

[8] Michael Pan, Sheng- Yan Chuang and Sheng -De Wang, "Local Repair Mechanisms for On - Demand Routing in Mobile Ad hoc Networks"

[9] Kishan Singh Rao and Laxmi Shrivastav," Efficient Local Route Repair Method in AODV to Reduce Congestion in Manet",Corona Journal of Science and Technology, pp 3538, Vol. 1 No. 1, Octuber 2012

[10] N.Javaid et al,"Modelling and Evaluating Enhancements in Expanding Ring Search Algorithm for Wireless Reactive Protocols", IEEE, April 2012

[11] Lei Guo, Yuhuai Peng, Xingwei Wang, Dingde Jiang and Yinpeng $\mathrm{Yu}$, "Performance evaluation for on-demand routing protocols based on OPNET modules in Wireless Mesh Networks", Computers and Electrical Engineering ,pp 106114,2011 